\title{
ON THE LITTLEWOOD PROBLEM MODULO A PRIME
}

\author{
BEN GREEN AND SERGEI KONYAGIN
}

Abstract. Let $p$ be a prime, and let $f: \mathbb{Z} / p \mathbb{Z} \rightarrow \mathbb{R}$ be a function with $\mathbb{E} f=0$ and $\|\widehat{f}\|_{1} \leqslant 1$. Then

$$
\min _{x \in \mathbb{Z} / p \mathbb{Z}}|f(x)|=O(\log p)^{-1 / 3+\epsilon} .
$$

One should think of $f$ as being "approximately continuous"; our result is then an "approximate intermediate value theorem".

As an immediate consequence we show that if $A \subseteq \mathbb{Z} / p \mathbb{Z}$ is a set of cardinality $\lfloor p / 2\rfloor$ then $\sum_{r}\left|\widehat{1_{A}}(r)\right| \gg(\log p)^{1 / 3-\epsilon}$. This gives a result on a "mod $p$ " analogue of Littlewood's well-known problem concerning the smallest possible $L^{1}$-norm of the Fourier transform of a set of $n$ integers.

Another application is to answer a question of Gowers. If $A \subseteq \mathbb{Z} / p \mathbb{Z}$ is a set of size $\lfloor p / 2\rfloor$ then there is some $x \in \mathbb{Z} / p \mathbb{Z}$ such that

$$
|| A \cap(A+x)|-p / 4|=o(p) .
$$

\section{INTRODUCTION}

The problem of Littlewood to which the title refers is the following question. If $A \subseteq \mathbb{Z}$ is a set of $n$ integers, what is the smallest possible value of the $L^{1}$-norm of the exponential sum over $A$. That is to say, what is

$$
I(n):=\min _{A \subseteq \mathbb{Z},|A|=n} \int_{0}^{1}\left|\sum_{n \in A} e^{2 \pi i n \theta}\right| d \theta ?
$$

The problem of determining the exact minimum, known as the strong Littlewood conjecture, is still unresolved (the conjecture is that the minimum occurs when $A$ is an arithmetic progression). However the bound $I(n) \gg \log n$, which is tight up to a constant factor, was obtained independently by the second-named author 8 ] and by McGehee, Pigno and Smith [10] some 20 years ago.

There are various natural variants of the problem that one might consider. In this paper we will be interested in the discrete setting of functions defined on the group $\mathbb{Z} / p \mathbb{Z}$. If $f: \mathbb{Z} / p \mathbb{Z} \rightarrow \mathbb{R}$ is a function, then instead of discussing exponential sums we speak of the Fourier transform of $f$. This is defined for $r \in \mathbb{Z} / p \mathbb{Z}$ by

$$
\widehat{f}(r):=\mathbb{E}_{x \in \mathbb{Z} / p \mathbb{Z}} f(x) e(r x / p) .
$$

This research was started when the authors visited PIMS, Vancouver, in Spring 2004. It was partially conducted during the period the first-named author served as a Clay Research Fellow. He would like to express his sincere gratitude to the Clay In stitute, and also to the Massachusetts Institute of Technology, where he was a Visiting Professor for the academic year 2005-06. The second-named author was supported by the INTAS grant 03-51-5070. 
Here $e(\theta)=e^{2 \pi i \theta}$, as is customary, and $\mathbb{E}$ denotes the averaging operator, which in this

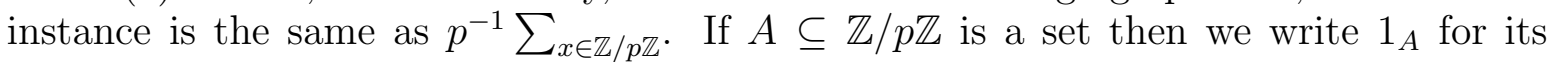
characteristic function. We will often be concerned with the Fourier transform $\widehat{1}_{A}$.

Question 1.1. Let $p$ be a prime, and let $A \subseteq \mathbb{Z} / p \mathbb{Z}$ be a set of size $\lfloor p / 2\rfloor$. What is $S(p)$, the minimum possible value of

$$
\left\|\widehat{1}_{A}\right\|_{1}:=\sum_{r \in \mathbb{Z} / p \mathbb{Z}}\left|\widehat{1}_{A}(r)\right| ?
$$

There is no serious reason for restricting attention to sets of size exactly $\lfloor p / 2\rfloor$, though clearly if sets of any size are allowed then $S(p)$ can equal 1. An obvious lower bound for $S(p)$ is $p^{-1}\lfloor p / 2\rfloor=1 / 2+O\left(p^{-1}\right)$, since this is the contribution to $\left\|\widehat{1_{A}}\right\|_{1}$ from the term $r=0$. It is already non-trivial to obtain a bound of the form $S(p) \rightarrow \infty$. We will obtain a very weak bound of this sort in $\$ 2$, followed in $\$$ 团 by the following stronger result.

Theorem 1.2. We have $S(p) \gg(\log p / \log \log p)^{1 / 3}$.

The best upper bound we have comes by considering the set $A=\{1, \ldots,\lfloor p / 2\rfloor\}$, which illustrates that $S(p) \ll \log p$. By analogy with the strong Littlewood conjecture, one might guess that this represents the truth.

Theorem 1.2 will be obtained as a straightforward consequence of the next result, which is the main theorem in our paper.

Theorem 1.3. Let $f: \mathbb{Z} / p \mathbb{Z} \rightarrow \mathbb{R}$ be a function with $\mathbb{E} f=0$ and $\|\widehat{f}\|_{1} \leqslant 1$. Then

$$
\min _{x \in \mathbb{Z} / p \mathbb{Z}}|f(x)|=O\left((\log \log p / \log p)^{1 / 3}\right) .
$$

The condition $\|\widehat{f}\|_{1} \leqslant 1$ may be thought of as expressing a (rather strong) kind of "continuity" or "smoothness" of $f$. Indeed one knows from harmonic analysis on the circle that if $f: \mathbb{R} / \mathbb{Z} \rightarrow \mathbb{R}$ is twice continuously differentiable then $\widehat{f}(m) \ll|m|^{-2}$ for $m \in \mathbb{Z} \backslash\{0\}$, and hence $\widehat{f}$ lies in $l^{1}(\mathbb{Z})$.

The conclusion of Theorem 1.3 may be thought of as a type of intermediate value theorem.

Taking $f(x)=c p /(p+1) \log p$ for $x=0,1, \ldots,\lfloor p / 2\rfloor$ and $f(x)=-c p /(p-1) \log p$ for $x=-1, \ldots,-\lfloor p / 2\rfloor$, where $c$ is a suitably small constant, one sees that the bound in Theorem 1.3 could not be improved beyond $O(1 / \log p)$. It may be that this is best possible.

As a corollary of Theorem 1.3] we are able to obtain some information on a question of Gowers, the consideration of which was in fact the starting point of our investigations. Gowers asked whether, if $A \subseteq \mathbb{Z} / p \mathbb{Z}$ is a set of size $\lfloor p / 2\rfloor$, there is some $x \in \mathbb{Z} / p \mathbb{Z}$ such that

$$
|| A \cap(A+x)|-p / 4|=o(p) .
$$

Note that $p / 4$ is roughly the expected value of $|A \cap(A+x)|$. One interesting feature of this question, and of Theorem [1.3] is that similar statements do not hold in abelian groups $G$ more general than $\mathbb{Z} / p \mathbb{Z}$; for example if $G=\mathbb{F}_{2}^{m}$ and if $A$ is a hyperplane then 
clearly $|A \cap(A+x)|$ is always either 0 or $|G| / 2$. We answer a generalisation of Gowers' question in the affirmative, obtaining as a consequence of Theorem 1.3 the following quantitative bound.

Theorem 1.4. Let $A, B \subseteq \mathbb{Z} / p \mathbb{Z}$ be subsets of $\mathbb{Z} / p \mathbb{Z}$ with $|A|=\alpha p,|B|=\beta p$. Then there is some $x \in \mathbb{Z} / p \mathbb{Z}$ such that

$$
|| A \cap(B+x)|-\alpha \beta p|=O\left(p(\log \log p / \log p)^{1 / 3}\right) .
$$

If $f, g: \mathbb{Z} / p \mathbb{Z} \rightarrow \mathbb{R}$ are two functions then we define their convolution $f * g$ by

$$
f * g(x):=\mathbb{E}_{y} f(y) g(x-y) .
$$

Note that

$$
|A \cap(B+x)|=p 1_{A} * 1_{B}^{\circ}(x),
$$

where we have written $f^{\circ}(x)=f(-x)$. It is thus easy to see that Theorem 1.4 is a special case of the following result:

Theorem 1.5. Let $f, g: \mathbb{Z} / p \mathbb{Z} \rightarrow \mathbb{R}$ be two functions with $\|f\|_{2},\|g\|_{2} \leqslant 1$. Then there is some $x \in \mathbb{Z} / p \mathbb{Z}$ such that

$$
|f * g(x)-\mathbb{E} f \mathbb{E} g|=O(\log \log p / \log p)^{1 / 3} .
$$

For Gowers' original problem it is not clear how much one can hope for, since there are no obvious examples of sets $A$ for which $|A \cap(A+x)|$ is always quite different to $p / 4$.

In $\$ 5$ and in $\$ 6$ we prove the following results.

Theorem 1.6. For any prime $p$ there is a function $f: \mathbb{Z} / p \mathbb{Z} \rightarrow \mathbb{R}$ such that $\mathbb{E} f=0$, $\|f\|_{2} \leqslant 1$, and

$$
\left|f * f^{\circ}(x)\right| \geqslant c / \log p
$$

for all $x \in \mathbb{Z} / p \mathbb{Z}$.

Theorem 1.7. For any prime $p \geqslant 3$ there is a set $A \subseteq \mathbb{Z} / p \mathbb{Z},|A|=\lfloor p / 2\rfloor$, such that

$$
|A \cap(A+x)-p / 4| \geqslant c p / \log p \log \log p
$$

for all $x \in \mathbb{Z} / p \mathbb{Z}$.

It would be of interest to close the gap between Theorem 1.4 and 1.7 and also to obtain the correct bound in Theorem 1.3

\section{The continuity argument, And a simple lower Bound}

The goal of this section is to motivate our main argument, and in so doing to prove a weak version of Theorem 1.3.

Theorem 2.1. Let $f: \mathbb{Z} / p \mathbb{Z} \rightarrow \mathbb{R}$ be a function with $\mathbb{E} f=0$ and $\|\widehat{f}\|_{1} \leqslant 1$. Then

$$
\min _{x \in \mathbb{Z} / p \mathbb{Z}}|f(x)|=o(1) .
$$

By the Fourier inversion formula we have

$$
f(x)=\sum_{r \in \mathbb{Z} / p \mathbb{Z}} \widehat{f}(r) e(-r x / p) .
$$


Since $\|\widehat{f}\|_{1}$ is so small, one might hope to have some rather direct control on this expression. Let us imagine, for a moment, that we know something even stronger about $\widehat{f}$, namely that for some fairly small set $R \subseteq \mathbb{Z} / p \mathbb{Z}$ we have

$$
\sum_{r \notin R}|\widehat{f}(r)| \leqslant \epsilon / 3
$$

By a well-known pigeonhole argument of Dirichlet, to be recalled in \$3, we may find some $t \neq 0$ such that the fractional parts $\{t r / p\}, r \in R$, are all small (we define our fractional parts to lie between $-1 / 2$ and $1 / 2)$. This implies that $|e(t r / p)-1|$ is always at most $\epsilon / 3$, say. Thus we have

$$
\begin{aligned}
|f(x)-f(x+t)| & =\sum_{r \in \mathbb{Z} / p \mathbb{Z}} \widehat{f}(r)(e(r x / p)-e(r(x+t) / p)) \\
& \leqslant \sum_{r \in R}|\widehat{f}(r)||1-e(r t / p)|+2 \sum_{r \notin R}|\widehat{f}(r)| \\
& \leqslant \epsilon / 3+2 \epsilon / 3=\epsilon
\end{aligned}
$$

for all $x$. Since $\mathbb{E} f(x)=0$, there must be some value of $x$ for which $f(x)$ and $f(x+t)$ have opposing signs. For this value of $x$ it is clear that $|f(x)| \leqslant \epsilon$.

Remark. What we have done here is identify a length, $t$, along which $f$ is somewhat continuous in the sense that $|f(x+t)-f(x)|$ is always small.

We now proceed to show how the above argument may be modified to give a weak version of Theorem 1.3. It need not be the case that most of the $L^{1}$-norm of $\widehat{f}$ is concentrated on a few modes, but by using a certain pigeonholing argument one may select a set $R$ of modes so that the contribution of $\sum_{r \notin R} \widehat{f}(r) e(-r x / p)$ is small for many $x$.

Let $\epsilon>0$, and define a sequence $\left(\delta_{j}\right)_{j=0}^{\infty}$ by $\delta_{0}=1$ and $\delta_{l+1}=\epsilon^{2+1 / \delta_{l}} 2^{-6 / \delta_{l}-4}$ for $l \geqslant 0$. Take $J=\lceil 3 / \epsilon\rceil$. Then there exists $l \in\{0, \ldots, J\}$ such that

$$
\sum_{r: \delta_{l+1} \leqslant|\widehat{f}(r)|<\delta_{l}}|\widehat{f}(r)| \leqslant \epsilon / 3 .
$$

For this value of $l$, define

$$
\begin{gathered}
f^{(1)}(x)=\sum_{r:|\widehat{f}(r)| \geqslant \delta_{l}} \widehat{f}(r) e(-r x / p), \\
f^{(2)}(x)=\sum_{r: \delta_{l+1} \leqslant|\widehat{f}(r)|<\delta_{l}} \widehat{f}(r) e(-r x / p)
\end{gathered}
$$

and

$$
f^{(3)}(x)=\sum_{r:|\widehat{f}(r)|<\delta_{l+1}} \widehat{f}(r) e(-r x / p) .
$$

By construction we have the estimate

$$
\left\|f^{(2)}\right\|_{\infty} \leqslant \epsilon / 3
$$


and also the bound

$$
\left\|f^{(3)}\right\|_{2}^{2}=\sum_{r:|\widehat{f}(r)|<\delta_{l+1}}|\widehat{f}(r)|^{2} \leqslant \delta_{l+1} .
$$

Write $R$ for the set of frequencies occurring in $f^{(1)}$, that is to say $R:=\left\{r:|\widehat{f}(r)| \geqslant \delta_{l}\right\}$, and set $k:=|R|$. By the assumption on $\|\widehat{f}\|_{1}$ we have $k \leqslant 1 / \delta_{l}$. Consider the Bohr set

$$
B(R, \eta):=\{x \in \mathbb{Z} / p \mathbb{Z}:|\{x r / p\}| \leqslant \eta \text { for all } r \in R\} .
$$

By Dirichlet's argument we have the bound

$$
|B(R, \eta)| \geqslant p \eta^{k} \geqslant p \eta^{1 / \delta_{l}}
$$

For details, and more properties of Bohr sets, see 93 (particularly Lemma 3.1 (i) and its proof).

Let $B:=B(R, \epsilon / 48)$. Suppose that $B \backslash\{0\}$ is nonempty, and select an element $t$ from it. By the same argument we had earlier, we have

$$
\left|f^{(1)}(x)-f^{(1)}(x+t)\right| \leqslant \sum_{r \in R}\left|\widehat{f^{(1)}}(r)\right||1-e(r t / p)| \leqslant \epsilon / 6 .
$$

Once again, since $\mathbb{E} f^{(1)}(x)=0$, there is $x_{0}$ such that $f^{(1)}\left(x_{0}\right)$ and $f^{(1)}\left(x_{0}+t\right)$ have opposing signs, which implies that $\left|f^{(1)}\left(x_{0}\right)\right| \leqslant \epsilon / 6$. For any $t \in B$ we then have $\left|f^{(1)}\left(x_{0}+t\right)\right| \leqslant \epsilon / 3$.

Set $X:=x_{0}+B$. Combining the above observations with (2.1) we have, if $x \in X$, the inequality

$$
|f(x)| \leqslant 2 \epsilon / 3+\left|f^{(3)}(x)\right|
$$

To conclude the argument it remains to show that there is some $x \in X$ for which $\left|f^{(3)}(x)\right| \leqslant \epsilon / 3$. To this end, note that if this were not the case then we should have

$$
\left\|f^{(3)}\right\|_{2}^{2}=\mathbb{E}_{x \in \mathbb{Z} / p \mathbb{Z}} f^{(3)}(x)^{2} \geqslant \frac{|B|}{p} \mathbb{E}_{x \in X} f^{(3)}(x)^{2}>|B| \epsilon^{2} / 9 \geqslant \epsilon^{2+1 / \delta_{l}} 2^{-6 / \delta_{l}-4} p,
$$

which contradicts (2.2).

The only condition that must be satisfied to make this argument work is that $B \backslash\{0\}$ must be non-empty. By dint of (2.3) this will be so if $p(\epsilon / 48)^{1 / \delta_{J}}>1$. Since $\delta_{J}$ is subject to a lower bound depending only on $\epsilon$, this can certainly be satisfied with $\epsilon=o(1)$, and in fact one can take $3 / \epsilon=\log _{*}(p)+O(1)$. This is a very slowly growing function, of course: the reason for this is that $1 / \delta_{J}$ looks like a tower of exponentials of height about $J$, this being forced by the need to have $\delta_{j+1}$ exponentially smaller than $\delta_{j}$.

Before moving on, let us highlight the weakness in the above argument which we will be able to tighten up in subsequent sections, leading to a superior bound. It lies in the fact that (2.4) represents not just a lower bound for $\left\|f^{(3)}\right\|_{2}^{2}$, but in fact for $\mathbb{E}_{x \in X}\left|f^{(3)}(x)\right|^{2}$. In order to exploit this properly one needs to modify (2.2) appropriately. Writing $\beta(x):=1_{B}(x) /|B|$ for the normalised Bohr cutoff associated to $B$, our interest lies in finding a Fourier expression for

$$
\mathbb{E}_{x \in \mathbb{Z} / p \mathbb{Z}}\left|f^{(3)}(x)\right|^{2} \beta\left(x+x_{0}\right)
$$


This is not hard; indeed we have

$$
\begin{aligned}
\mathbb{E}_{x}\left|f^{(3)}(x)\right|^{2} \beta\left(x+x_{0}\right) & =\mathbb{E}_{x} \sum_{r, r^{\prime}} \widehat{f^{(3)}}(r) \overline{\widehat{f^{(3)}}\left(r^{\prime}\right)} e\left(\left(r^{\prime}-r\right) x / p\right) \beta\left(x+x_{0}\right) \\
& =\sum_{r, r^{\prime}} \widehat{f^{(3)}}(r) \widehat{\widehat{f^{(3)}}\left(r^{\prime}\right)} \widehat{\beta}\left(r-r^{\prime}\right) e\left(\left(r-r^{\prime}\right) x_{0} / p\right),
\end{aligned}
$$

an expression we will find very useful later on. In exploiting it we will require a few facts concerning Bohr sets and their Fourier transforms. We develop these in the next section.

\section{Properties of Bohr sets}

The material of this section is somewhat technical, but is rapidly becoming part of the foundation of additive combinatorics. It has its origins in the work of Bourgain [2] on 3-term arithmetic progressions. See [12] for a recent exposition of this work. For examples of subsequent work in a similar vein, see either [5] or [13, and for a more leisurely discussion of Bohr sets and the need for the results of this section, see [6]. We work in the context of an arbitrary finite abelian group $G$, since this is most natural, though our interest is in the case $G=\mathbb{Z} / p \mathbb{Z}$. In fact, a lot of the material in this paper is valid in this more general setting, the key exception being the "discrete intermediate value theorem" of \$1 The failure of this, of course, means that the key theorems as stated in $\S \mathbb{1}$ are only valid when $G=\mathbb{Z} / p \mathbb{Z}$.

If $f: G \rightarrow \mathbb{R}$ is a function and if $\gamma \in G^{*}$ is a character then we define the Fourier transform $\widehat{f}$ by

$$
\widehat{f}(\gamma):=\mathbb{E}_{x \in G} f(x) \gamma(x) .
$$

It is easy to tie this in with the definition we had in the case $G=\mathbb{Z} / p \mathbb{Z}$; in that group, all characters are of the form $x \mapsto e(r x / p)$ for some $r \in \mathbb{Z} / p \mathbb{Z}$.

Now if $\Gamma \subseteq G^{*}$ is a set of $d$ characters, and if $\epsilon>0$, then we define the Bohr set $B=B(\Gamma, \epsilon)$ by

$$
B(\Gamma, \epsilon):=\left\{x \in G:\left|\frac{1}{2 \pi} \arg \gamma(x)\right| \leqslant \epsilon \text { for all } \gamma \in \Gamma\right\}
$$

We also define $\beta=\beta_{\Gamma, \epsilon}$ (the normalised Bohr cutoff associated to $B$ ) by $\beta(x):=$ $1_{B}(x) /|B|$.

The next lemma details some well-known facts concerning the size of Bohr sets.

Lemma 3.1. Let $\Gamma \subseteq G^{*}$ be a set of $d$ characters, and let $\delta>0$. Then we have the bounds

(i) $|B(\Gamma, \delta)| \geqslant \delta^{d}|G|$;

(ii) $|B(\Gamma, 2 \delta)| \leqslant 5^{d}|B(\Gamma, \delta)|$.

Proof. For any $\eta$, write $S_{\eta}$ for the set of all $y=\left(y_{1}, \ldots, y_{d}\right) \in \mathbb{R}^{d} / \mathbb{Z}^{d}$ for which $\left\|y_{j}\right\|_{\infty} \leqslant$ $\eta / 2$ for all $j=1, \ldots, d$ (to define the $\|\cdot\|_{\infty}$ norm on $\mathbb{R} / \mathbb{Z}$ we identify it with $\left(-\frac{1}{2}, \frac{1}{2}\right]$ ). Let $\Gamma=\left\{\left(\gamma_{1}, \ldots, \gamma_{d}\right)\right\}$. Now if $x \in G$ write

$$
v(x)=\frac{1}{2 \pi}\left(\arg \gamma_{1}(x), \ldots, \arg \gamma_{d}(x)\right) \in \mathbb{R}^{d} / \mathbb{Z}^{d} .
$$


If $v(x)$ and $v\left(x^{\prime}\right)$ both lie in some translate $a+S_{\delta}$ then $x-x^{\prime} \in B(\Gamma, \delta)$, and so for fixed $x^{\prime} \in G \cap v^{-1}\left(\left(a+S_{\delta}\right)\right)$ the map $x \mapsto x-x^{\prime}$ defines an injection from $G \cap v^{-1}\left(a+S_{\delta}\right)$ to $B(\Gamma, \delta)$. Hence

$$
\left|v(G) \cap\left(a+S_{\delta}\right)\right| \leqslant|B(\Gamma, \delta)|
$$

Proof of (i). By a simple averaging there is some translate $a+S_{\delta}$ such that

$$
\left|v(G) \cap\left(a+S_{\delta}\right)\right| \geqslant\left|S_{\delta}\right||v(G)|=\left|S_{\delta}\right|=\delta^{d}|G|
$$

The result is now immediate from (3.1).

Proof of (ii). From (3.1) one has

$$
\left|v(B(\Gamma, 2 \delta)) \cap\left(a+S_{\delta}\right)\right| \leqslant\left|v(G) \cap\left(a+S_{\delta}\right)\right| \leqslant|B(\Gamma, \delta)| .
$$

Now $v(B(\Gamma, 2 \delta)) \cap\left(a+S_{\delta}\right)$ is empty unless $a \in S_{5 \delta}$, and so

$|B(\Gamma, 2 \delta)|=\frac{1}{\left|S_{\delta}\right|} \int_{\mathbb{R}^{d} / \mathbb{Z}^{d}}\left|v(B(\Gamma, 2 \delta)) \cap\left(a+S_{\delta}\right)\right| d a \leqslant|B(\Gamma, \delta)| \cdot \frac{\left|S_{5 \delta}\right|}{\left|S_{\delta}\right|}=5^{d} \cdot|B(\Gamma, \delta)|$.

Suppose now that $x \in B(\Gamma, \epsilon)$ and that $x^{\prime} \in B\left(\Gamma, \epsilon^{\prime}\right)$, where $\epsilon^{\prime} \ll \epsilon$. Then $x+x^{\prime} \in$ $B\left(\Gamma, \epsilon+\epsilon^{\prime}\right)$. Assuming that $B\left(\Gamma, \epsilon+\epsilon^{\prime}\right)$ is not much bigger than $B(\Gamma, \epsilon)$, this means that $B(\Gamma, \epsilon)+B\left(\Gamma, \epsilon^{\prime}\right) \approx B(\Gamma, \epsilon)$. After passing to the associated normalised cutoff functions $\beta$ and $\beta^{\prime}$, we might anticipate that $\beta * \beta^{\prime} \approx \beta$. When true this property, which says that Bohr cutoffs are roughly invariant under convolution by narrower Bohr cutoffs, is extremely useful.

Unfortunately the property does not always hold, since the size of $B(\Gamma, t)$ need not vary very smoothly with $t$. For example, suppose that $G=\mathbb{F}_{5}^{n}$ and that $\delta_{1}<1 / 5<\delta_{2}$. Then if $|\Gamma|$ consists of $d$ linearly independent characters we have $\left|B\left(\Gamma, \delta_{1}\right)\right|=5^{-d}|G|$ whilst $\left|B\left(\Gamma, \delta_{2}\right)\right|=(3 / 5)^{d}|G|$.

Bourgain showed, and we shall repeat his argument, that for a fixed $\Gamma$ there are a plentiful supply of $\epsilon$ such that the function $|B(\Gamma, t)|$ does vary quite regularly for $t \approx \epsilon$, in the following sense.

Definition 3.2 (Regular value). Let $\Gamma$ be a set of $d$ characters. We say that $B(\Gamma, \epsilon)$ is regular value, or that $\epsilon$ is a regular value for $\Gamma$, if

$$
1-100 d|\kappa| \leqslant \frac{|B(\Gamma,(1+\kappa) \epsilon)|}{|B(\Gamma, \epsilon)|} \leqslant 1+100 d|\kappa|
$$

whenever $|\kappa| \leqslant 1 / 100 d$.

Proposition 3.3. Let $\Gamma,|\Gamma|=d$, be a fixed set of characters. Let $\delta \in(0,1)$. Then there is $\epsilon \in[\delta, 2 \delta)$ which is regular for $\Gamma$.

Proof. Set $f(\alpha)=|B(\Gamma, 2 \alpha \delta)|$. Then $f(\alpha)$ is a non-decreasing function on $\left[\frac{1}{2}, 1\right]$ and, by Lemma 3.1 .

$$
f(1) \leqslant 5^{d} f(1 / 2)
$$

We wish to show that there is $\alpha \in[1 / 2,1]$ such that $1-100 d|\kappa| \leqslant f((1+\kappa) \alpha) / f(\alpha) \leqslant$ $1+100 d|\kappa|$ for all $|\kappa| \leqslant 1 / 100 d$. Suppose then that this is false. Observe that $\frac{1}{1-x} \geqslant 1+x$ 
when $x \geqslant 0$; hence for every $\alpha \in[1 / 2,1]$ there is $t_{\alpha} \in[0,1 / 100 d]$ such that

$$
\left|\frac{f\left(\left(1+t_{\alpha}\right) \alpha\right)}{f\left(\left(1-t_{\alpha}\right) \alpha\right)}\right| \geqslant 1+100 d t_{\alpha} \geqslant e^{50 d t_{\alpha}}
$$

the last step following because $1+x \geqslant e^{x / 2}$ for $x \leqslant 1$.

The next lemma is related to the Vitali covering lemma, but is so simple that we provide an independent proof. For an anecdotal discussion see [3].

Lemma 3.4. Suppose a finite collection of closed intervals $I_{1}, \ldots, I_{k}$ covers $[0,1]$. Then we can pick a subcollection $I_{i_{1}}, \ldots, I_{i_{m}}$ whose members are disjoint except possibly at their endpoints, with total measure at least $1 / 2$.

Proof. Without loss of generality suppose that the collection $I_{1}, \ldots, I_{k}$ is minimal in that if any $I_{j}$ is removed, the intervals no longer cover $[0,1]$. It is then easy to see that no point $x$ lies in three of the $I_{j}$, because there are two in tervals $I_{r}$ and $I_{s}$ containing $x$ such that any other $I_{t}$ containing $x$ lies in $I_{r} \cup I_{s}$. But it is then easy to describe the form of the intervals exactly. Suppose that the $I_{j}=\left[a_{j}, b_{j}\right]$ with $a_{1} \leqslant a_{2} \leqslant \ldots \leqslant a_{k}$. Then

$$
a_{1} \leqslant a_{2} \leqslant b_{1} \leqslant a_{3} \leqslant b_{2} \leqslant a_{4} \leqslant \ldots \leqslant b_{k-1} \leqslant b_{k} .
$$

It follows that the two collections $I_{1} \cup I_{3} \cup \ldots$ and $I_{2} \cup I_{4} \cup \ldots$ contain disjoint intervals. The result is now obvious.

To apply Lemma 3.4 recall (3.3). By compactness we may take a finite set

$$
\left\{\alpha_{1}, \ldots, \alpha_{k}\right\} \subseteq\left[\frac{1}{2}+\frac{1}{100 d}, 1-\frac{1}{100 d}\right]
$$

such that the intervals $\left[\left(1-t_{\alpha_{i}}\right) \alpha_{i},\left(1+t_{\alpha_{i}}\right) \alpha_{i}\right]$ cover $\left[\frac{1}{2}+\frac{1}{100 d}, 1-\frac{1}{100 d}\right]$. Since $t_{\alpha_{i}} \leqslant$ $1 / 100 d$, all of these intervals are contained in $\left[\frac{1}{2}, 1\right]$. By Lemma 3.4, we can pick a disjoint subcollection of measure at least $\frac{1}{4}(1-1 / 100 d)>\frac{1}{5}$. Letting these intervals correspond to $\left\{\alpha_{1}, \ldots, \alpha_{l}\right\}$, one has $2 \sum_{i=1}^{l} \alpha_{i} t_{\alpha_{i}}>1 / 5$, and so $\sum_{i=1}^{l} t_{\alpha_{i}}>1 / 10$. Using this in (3.3) gives

$$
\prod_{i=1}^{l}\left|\frac{f\left(\left(1+t_{\alpha_{i}}\right) \alpha_{i}\right)}{f\left(\left(1-t_{\alpha_{i}}\right) \alpha_{i}\right)}\right| \geqslant e^{50 d \sum_{i} t_{\alpha_{i}}}>e^{5 d} .
$$

However the left hand side is at most $f(1) / f(1 / 2)$, and hence by (3.2) we have

$$
5^{d}>e^{5 d}
$$

This is a contradiction, and Proposition 3.3 is established.

Remark. Actually, Proposition 3.3 easily follows from weak type estimates for the Hardy-Littlewood maximal function. Let $\nu$ be a bounded non-decreasing function $\mathbb{R} \rightarrow$ $\mathbb{R}$ with an associated Radon-Stieltjes measure

$$
\nu[a, b)=\nu(b)-\nu(a) \quad(a<b) .
$$

Note that

$$
\nu(\mathbb{R})=\lim _{b \rightarrow \infty} \nu[-b, b) .
$$

The Hardy-Littlewood maximal function is defined as

$$
M_{\nu}(t)=\sup _{a<t<b} \frac{\nu(b)-\nu(a)}{b-a} .
$$


The weak type estimates for $M_{n}(u)$ claim that for any $\alpha>0$

$$
\mu\left\{t: M_{\nu}(t)>\alpha\right\} \leq \frac{2 \nu(\mathbb{R})}{\alpha}
$$

where $\mu$ is the Lebesgue measure. For an absolutely continuous $\nu$ this inequality is classical; a general case was established in [9]. One can prove [3.3 applying (3.4) to the function $\nu$ such that $\nu(x)=\log f\left(e^{x}\right)$ for $x \in[-\log 2,0], \nu(x)=\nu(-\log 2)$ for $x<-\log 2$, and $\nu(x)=\nu(0)$ for $x>0$.

Lemma 3.5. Let $\Gamma \subseteq G^{*}$ be a set of $d$ characters, and let $\delta, \epsilon>0$. Suppose that $\eta$ is a regular value for $\Gamma$, and let $\beta=\beta_{\Gamma, \eta}$ be the normalised Bohr cutoff associated to $B(\Gamma, \eta)$. Suppose that $y \in B\left(\Gamma, \eta^{\prime}\right)$, where $\eta^{\prime} \leqslant \epsilon \eta / 200 d$. Then

$$
\mathbb{E}_{x \in G}|\beta(x+y)-\beta(x)| \leqslant \epsilon .
$$

Proof. Set $\delta:=\epsilon / 200 d$. If $\beta(x+y)-\beta(x) \neq 0$ then $x \in B(\Gamma, \eta(1+\delta)) \backslash B(\Gamma, \eta(1-\delta))$. Thus, since $B=B(\Gamma, \eta)$ is regular, we have

$$
\sum_{x}\left|1_{B}(x+y)-1_{B}(x)\right| \leqslant 200 d \delta|B|,
$$

which immediately implies the result.

The following corollary will be very useful. It tells us that if $\widehat{\beta}(\gamma)$ is moderately large, then $\gamma(y) \approx 1$ for $y$ in a smaller Bohr set $B\left(\Gamma, \eta^{\prime}\right)$.

Lemma 3.6. Let $B(\Gamma, \eta)$ be a regular Bohr set with normalised cutoff $\beta=\beta_{\Gamma, \eta}$. Suppose that $\kappa_{1}, \kappa_{2}>0$. Suppose that $\gamma$ is a character for which $|\widehat{\beta}(\gamma)| \geqslant \kappa_{1}$, and that $y \in$ $B\left(\Gamma, \eta^{\prime}\right)$, where $\eta^{\prime} \leqslant \kappa_{1} \kappa_{2} \eta / 200 d$. Then $|1-\gamma(y)| \leqslant \kappa_{2}$.

Proof. We have

$$
\kappa_{1}|1-\gamma(y)| \leqslant|\widehat{\beta}(\gamma)||1-\overline{\gamma(y)}|=\left|\mathbb{E}_{x \in G}(\beta(x+y)-\beta(x)) \gamma(x)\right|,
$$

which is at most $\kappa_{1} \kappa_{2}$ by Lemma 3.5. The result follows.

Remark. By employing appropriate smoothing devices, one may obtain a reasonably good quantitative description of the set of $\gamma$ for which $\left|\widehat{\beta_{\Gamma, \epsilon}}(\gamma)\right| \geqslant \delta$ when $B(\Gamma, \epsilon)$ is regular. If $\Gamma=\left\{\gamma_{1}, \ldots, \gamma_{d}\right\}$ these characters will all be of the form $\gamma_{1}^{a_{1}} \ldots \gamma_{d}^{a_{d}}$, where the $a_{i}$ are integers bounded in absolute value by some $F(\delta)$. We will not need to use such information in this paper.

\section{Proofs of the MAIN THEOREMS}

Our first task in this section will be to supply a proof of Theorem 1.3. We begin with some motivating remarks which, it will turn out, take us some distance into the proof itself. Let $f: \mathbb{Z} / p \mathbb{Z} \rightarrow \mathbb{R}$ be a function with $\|\widehat{f}\|_{1} \leqslant 1$, as in the statement of that theorem. Suppose to begin with that we have defined a splitting $f=f^{(1)}+f^{(2)}$ where

$$
f^{(1)}(x):=\sum_{\gamma \in \Gamma} \widehat{f}(\gamma) \overline{\gamma(x)}
$$


for some reasonably small set $\Gamma$. If $f^{(1)}\left(x_{0}\right)$ is small then $f^{(1)}\left(x+x_{0}\right)$ will also be small provided $x \in B(\Gamma, \eta)$, for suitably small $\eta$. Our first observation is that much the same would be true if $\Gamma=\left\{\gamma_{1}, \ldots, \gamma_{d}\right\}$ were replaced by some set

$$
\left\{\gamma_{1}^{a_{1}} \ldots \gamma_{d}^{a_{d}}:\left|a_{i}\right| \leqslant M\right\}
$$

consisting of small combinations of elements of $\Gamma$. By remarks made at the end of 33 , the characteristic function of this set rather resembles the function $\widehat{\beta_{\Gamma, \eta}}$, for suitable $\eta$. Once this is noticed, one might think to redefine

$$
f^{(1)}:=\sum_{\gamma} \widehat{\beta_{\Gamma, \eta}}(\gamma) \widehat{f}(\gamma) \overline{\gamma(x)}=f * \beta_{\Gamma, \eta},
$$

which looks extremely natural. To begin one takes $\Gamma=\emptyset$; we add frequencies according to an iterative procedure.

Lemma 4.1. Suppose that $B(\Gamma, \eta)$ is regular, that $\left|f^{(1)}\left(x_{0}\right)\right| \leqslant \epsilon$, and that $x \in B\left(\Gamma, \eta^{\prime}\right)$ for some $\eta^{\prime} \leqslant \epsilon^{2} \eta / 200 d$. Then $\left|f^{(1)}\left(x+x_{0}\right)\right| \leqslant 3 \epsilon$.

Proof. We have

$$
\left|f^{(1)}\left(x+x_{0}\right)-f^{(1)}\left(x_{0}\right)\right|=\left|\sum_{\gamma} \widehat{f}(\gamma) \widehat{\beta_{\Gamma, \eta}}(\gamma) \gamma\left(x_{0}\right)(\gamma(x)-1)\right| \leqslant \sup _{\gamma}\left|\widehat{\beta_{\Gamma, \eta}}(\gamma)\right||1-\gamma(x)| .
$$

Now if $\left|\widehat{\beta_{\Gamma, \eta}}(\gamma)\right| \leqslant \epsilon$ then clearly

$$
\left|\widehat{\beta_{\Gamma, \eta}}(\gamma)\right||1-\gamma(x)| \leqslant 2 \epsilon
$$

If, however, $\left|\widehat{\beta_{\Gamma, \eta}}(\gamma)\right| \geqslant \epsilon$ and if $x \in B\left(\Gamma, \eta^{\prime}\right)$ then Lemma 3.6 tells us that $|1-\gamma(x)| \leqslant \epsilon$, and so (4.1) holds in this case too.

Now define

$$
f^{(2)}:=f-f^{(1)} .
$$

Suppose that $f^{(1)}\left(x_{0}\right)$ is small. By Lemma 4.1 we know that $f^{(1)}\left(x+x_{0}\right)$ is small for all $x \in B\left(R, \eta^{\prime}\right)$, provided $\eta^{\prime}$ is not too large. Our hope is that $f^{(1)}\left(x+x_{0}\right)$ will also be small for some such $x$. To this end we look at

$$
E:=\mathbb{E}_{x \in G}\left|f^{(2)}\left(x+x_{0}\right)\right|^{2} \beta_{\Gamma, \eta^{\prime}}(x) \leqslant \sum_{\gamma, \gamma^{\prime}}\left|\widehat{f^{(2)}}(\gamma)\right|\left|\widehat{f^{(2)}}\left(\gamma^{\prime}\right)\right|\left|\widehat{\beta_{\Gamma, \eta}}\left(\gamma-\gamma^{\prime}\right)\right|,
$$

the inequality being an immediate consequence of (2.5). Write

$$
U:=\left\{\chi:\left|\widehat{\beta_{\Gamma, \eta^{\prime}}}(\chi)\right| \geqslant \epsilon^{2}\right\} .
$$

Observing that

$$
\sum_{\gamma}\left|\widehat{f^{(2)}}(\gamma)\right|=\sum_{\gamma}|\widehat{f}(\gamma)|\left|1-\widehat{\beta_{\Gamma, \eta}}(\gamma)\right| \leqslant\|\widehat{f}\|_{1} \sup _{\gamma}\left|1-\widehat{\beta_{\Gamma, \eta}}(\gamma)\right| \leqslant 2,
$$

it is immediate from (4.3) that

$$
E \leqslant 4 \epsilon^{2}+\sum_{\substack{\gamma, \gamma^{\prime} \\ \gamma-\gamma^{\prime} \in U}}\left|\widehat{f^{(2)}}(\gamma)\right|\left|\widehat{f^{(2)}}\left(\gamma^{\prime}\right)\right| \widehat{\beta_{\Gamma, \eta^{\prime}}}\left(\gamma-\gamma^{\prime}\right)\left|\leqslant 4 \epsilon^{2}+2 \sup _{\gamma} \sum_{\gamma^{\prime}: \gamma^{\prime}-\gamma \in U}\right| \widehat{f^{(2)}}\left(\gamma^{\prime}\right) \mid .
$$

Now if

$$
\sup _{\gamma} \sum_{\gamma^{\prime}: \gamma^{\prime}-\gamma \in U}\left|\widehat{f^{(2)}}\left(\gamma^{\prime}\right)\right| \leqslant \epsilon^{2}
$$


then $E \leqslant 6 \epsilon^{2}$, and there is indeed $x \in B\left(\Gamma, \eta^{\prime}\right)$ such that $\left|f^{(2)}\left(x+x_{0}\right)\right| \leqslant \epsilon \sqrt{6} \leqslant 3 \epsilon$. For this $x$, we have $\left|f\left(x+x_{0}\right)\right| \leqslant\left|f^{(1)}\left(x+x_{0}\right)\right|+\left|f^{(2)}\left(x+x_{0}\right)\right| \leqslant 6 \epsilon$.

If, on the other hand, there is an $\gamma$ such that

$$
\sum_{\gamma^{\prime}: \gamma^{\prime}-\gamma \in U}\left|\widehat{f^{(2)}}\left(\gamma^{\prime}\right)\right| \geqslant \epsilon^{2}
$$

then we must find an alternative argument. It is tempting to somehow "add" the offending characters $u+\gamma, u \in U$, into the definition of $f^{(1)}$. Thus we might consider a new set $\widetilde{\Gamma}:=\Gamma \cup\{\gamma\}$ and some $\widetilde{\eta} \ll \eta$ such that $\widehat{\beta_{\widetilde{\Gamma}, \widetilde{\eta}}}(s) \approx 1$ for $s=u+\gamma$.

Once this is done, one may iterate the whole procedure starting with (4.2). If things are done carefully, the obstacle (4.6) cannot be encountered more than $\epsilon^{-2}$ times since each new instance corresponds to at least $\epsilon^{2}$ of the total $L^{1}$-mass of $\widehat{f}$, which we are assuming is at most 1 . Care must be taken to ensure that these portions of $L^{1}$-mass are all disjoint, but we will see later on that this can be arranged.

Working out the details of the above argument, one obtains a bound $\min _{x}|f(x)|=$ $O\left((\log p)^{-1 / 4+\varepsilon}\right)$ in Theorem 1.3. To obtain the superior exponent $\frac{1}{3}$, we exploit the fact that it is not necessary for $f^{(2)}$ itself to satisfy (4.5); we may perturb $f^{(2)}$ by an arbitrary function $f^{(3)}$ for which $\left\|\widehat{f^{(3)}}\right\|_{1} \leqslant \epsilon$, since then

$$
\left|f^{(3)}(x)\right|=\left|\sum_{\gamma} \widehat{f^{(3)}}(\gamma) \overline{\gamma(x)}\right| \leqslant\left\|\widehat{f^{(3)}}\right\|_{1} \leqslant \epsilon
$$

for an arbitrary $x$. Thus in effect we wish to write

$$
f=f^{(1)}+f^{(2)}+f^{(3)},
$$

where $f^{(2)}$ satisfies (4.5) and

$$
\left\|\widehat{f^{(3)}}\right\|_{1} \leqslant \epsilon .
$$

This, it transpires, can be achieved by a slight variant of the above iterative scheme in which the number of iterations is reduced to just $\epsilon^{-1}$.

We now turn to the proof proper of Theorem 1.3, beginning with a lemma summarising the above discussion.

Lemma 4.2. Let $B=B(\Gamma, \eta)$ and $B^{\prime}=B\left(\Gamma, \eta^{\prime}\right)$ be regular Bohr sets with normalised Bohr cutoffs $\beta=\beta_{\Gamma, \eta}$ and $\beta^{\prime}=\beta_{\Gamma, \eta^{\prime}}$. Suppose that $\eta^{\prime} \leqslant \epsilon^{2} \eta / 200 d$. Set

$$
U:=\left\{\chi:\left|\widehat{\beta^{\prime}}(\chi)\right| \geqslant \epsilon^{2}\right\} .
$$

Suppose that $f=f^{(1)}+f^{(2)}+f^{(3)}$, where $f^{(1)}=f * \beta$, that

$$
\sup _{\gamma} \sum_{\gamma^{\prime}: \gamma^{\prime}-\gamma \in U}\left|\widehat{f^{(2)}}\left(\gamma^{\prime}\right)\right| \leqslant \epsilon^{2}
$$

and that $\left\|\widehat{f^{(3)}}\right\|_{1} \leqslant \epsilon$. Suppose that $\min _{x}\left|f^{(1)}(x)\right| \leqslant 2 \epsilon$. Then $\min |f(x)| \leqslant 8 \epsilon$.

Proof. Suppose that $\left|f^{(1)}\left(x_{0}\right)\right| \leqslant 2 \epsilon$. Then Lemma 4.1 tells us that for any $x \in B\left(R, \eta^{\prime}\right)$ we have $\left|f^{(1)}\left(x+x_{0}\right)\right| \leqslant 4 \epsilon$. Now by the discussion leading to (4.5) we know that 
there is $x \in B\left(R, \eta^{\prime}\right)$ for which $\left|f^{(2)}\left(x+x_{0}\right)\right| \leqslant 3 \epsilon$, and finally from (4.7) we have $\left|f^{(3)}\left(x+x_{0}\right)\right| \leqslant \epsilon$ for any $x$ whatsoever. The result follows.

The next lemma asserts that there is a decomposition of the type discussed in Lemma 4.2

Lemma 4.3. Let $\epsilon>0$. Then there is a set $\Gamma,|\Gamma| \leqslant 2 / \epsilon^{2}$, of characters, an $\eta \geqslant$ $\left(\epsilon^{9} / 2^{21}\right)^{2 / \epsilon}$ and an $\eta^{\prime} \geqslant 2^{-10} \epsilon^{3} \eta$ such that the hypotheses of Lemma 4.2 are satisfied, with the possible exception of the inequality $\min _{x}\left|f^{(1)}(x)\right| \leqslant \epsilon$.

Proof. We will have a kind of double iterative scheme, the STEPs of which will be indexed by pairs $(j, i)$. The two coordinates of STEP will be denoted $\mathrm{STEP}_{1}$ and $\mathrm{STEP}_{2}$. The algorithm will proceed by first incrementing $\mathrm{STEP}_{2}$, that is to say according to the following scheme:

$$
(0,0) \rightarrow(0,1) \rightarrow \cdots \rightarrow\left(0, l_{0}\right) \rightarrow(1,0) \rightarrow(1,1) \rightarrow \cdots \rightarrow\left(1, l_{1}\right) \rightarrow(2,0) \rightarrow \ldots
$$

Whilst $\mathrm{STEP}_{1}=j$ there will be a set $\Gamma_{j}$ of characters and regular Bohr sets $B\left(\Gamma_{j}, \eta_{j}\right)$, $B\left(\Gamma_{j}, \eta_{j}^{\prime}\right)$ with normalised Bohr cutoffs $\beta_{j}:=\beta_{\Gamma_{j}, \eta_{j}}$ and $\beta_{j}^{\prime}:=\beta_{\Gamma_{j}, \eta_{j}^{\prime}}$. The parameters $\eta_{j}$ and $\eta_{j}^{\prime}$ will be related by the inequalities

$$
\epsilon^{2} \eta_{j} / 400 d \leqslant \eta_{j}^{\prime} \leqslant \epsilon^{2} \eta_{j} / 200 d
$$

in order that we may apply Lemma 4.2. In fact $\eta_{j}^{\prime}$ can simply be an arbitrary parameter in this range for which $B\left(\Gamma_{j}, \eta_{j}^{\prime}\right)$ is regular. There will also be disjoint sets $S_{j, i}$ of characters, $i=1,2, \ldots$ When $\operatorname{STEP}=(j, i)$ we will have occasion to discuss the union

$$
\Omega_{j}:=\bigcup_{j^{\prime}<j} \bigcup_{i \leqslant l_{j^{\prime}}} S_{j^{\prime}, i}
$$

of all characters which were defined whilst $\operatorname{STEP}_{1}<j$. Things will have been arranged so that $B\left(\Gamma_{j}, \eta_{j}\right) \subseteq B\left(\Omega_{j}, \epsilon\right)$; the set $\Omega_{j}$ should be thought of as containing small combinations of elements of $\Gamma_{j}$, and in fact the proof could be set up in such a way that this is made explicit (see the remarks at the end of \$3). In proceeding from STEP $=(j, 0)$ to $\operatorname{STEP}=(j, i)$ we will have accrued a collection $\left\{\gamma_{j, 1}, \ldots, \gamma_{j, i}\right\}$ of characters; these will be used to define $\Gamma_{j+1}$.

To initialize the iteration set $\operatorname{STEP}=(0,0)$, and define $\Gamma_{0}=\emptyset$ and $\eta_{0}=1$. Now suppose that $\operatorname{STEP}=(j, i)$. Define

$$
f_{j}^{(1)}:=f * \beta_{j},
$$

and set $g_{j}:=f-f_{j}^{(1)}$.

Define $U_{j}:=\left\{s:\left|\widehat{\beta}_{j}^{\prime}(s)\right| \geqslant \epsilon^{2}\right\}$. We will have reached $\operatorname{STEP}=(j, i)$ by incrementing $\mathrm{STEP}_{2}$, starting from STEP $=(j, 0)$. During this process we will have defined disjoint sets $S_{j, 1}, S_{j, 2}, \ldots, S_{j, i}$ of frequencies, and also a collection $\left\{\gamma_{j, 1}, \ldots, \gamma_{j, i}\right\}$. The construction will be such that

$$
S_{j, i} \subseteq \gamma_{j, i}+U_{j}
$$

for all $i$. We begin by asking whether or not

$$
\sum_{\gamma^{\prime} \in S_{j, 1} \cup S_{j, 2} \cup \cdots \cup S_{j, i}}\left|\widehat{g_{j}}\left(\gamma^{\prime}\right)\right| \geqslant \epsilon .
$$


If so, it is time to increment $\operatorname{STEP}_{1}$ and to define $\Gamma_{j+1}$ and $\eta_{j+1}$. We set $\operatorname{STEP}=(j+1,0)$, $\Gamma_{j+1}:=\Gamma_{j} \cup\left\{\gamma_{j, 1}, \ldots, \gamma_{j, i}\right\}$ and choose $\eta_{j+1}$,

$$
\epsilon^{3} \eta_{j}^{\prime} / 800\left|\Gamma_{j}\right| \leqslant \eta_{j+1} \leqslant \epsilon^{3} \eta_{j}^{\prime} / 400\left|\Gamma_{j}\right|,
$$

in such a way that the Bohr set $B\left(\Gamma_{j+1}, \eta_{j+1}\right)$ is regular. Clearly $\eta_{j+1} \leqslant \min \left(\eta_{j}, \epsilon / 2\right)$. Note that

$$
\Omega_{j+1}=\Omega_{j} \cup S_{j, 1} \cup S_{j, 2} \cup \cdots \cup S_{j, i} .
$$

Claim 1. We have the inclusion

$$
B\left(\Gamma_{j+1}, \eta_{j+1}\right) \subseteq B\left(\Omega_{j+1}, \epsilon\right)
$$

Proof. Clearly

$$
B\left(\Gamma_{j+1}, \eta_{j+1}\right) \subseteq B\left(\Gamma_{j}, \eta_{j}\right) \subseteq B\left(\Omega_{j}, \epsilon\right) \subseteq B\left(\Omega_{j+1}, \epsilon\right),
$$

and so it suffices to check that $B\left(\Gamma_{j+1}, \eta_{j+1}\right) \subseteq B\left(S_{j, i}, \epsilon\right)$. For this, in view of (4.11), we only need confirm that

$$
B\left(\Gamma_{j+1}, \eta_{j+1}\right) \subseteq B\left(\gamma_{j, i}, \epsilon / 2\right) \cap B\left(U_{j}, \epsilon / 2\right)
$$

for each $i$. The inclusion $B\left(\Gamma_{j+1}, \eta_{j+1}\right) \subseteq B\left(\gamma_{j, i}, \epsilon / 2\right)$ is immediate from t he fact that $\eta_{j+1} \leqslant \epsilon / 2$. To see that $B\left(\Gamma_{j+1}, \eta_{j+1}\right) \subseteq B\left(U_{j}, \epsilon / 2\right)$, we use Lemma 3.6. Indeed if $\gamma \in U_{j}$ and if $y \in B\left(\Gamma_{j}, \eta_{j+1}\right)$ then by that lemma we have $|1-\gamma(y)| \leqslant \epsilon / 2$, which is exactly what we need.

Now if (4.12) does not hold then we carry on incrementing $\operatorname{STEP}_{2}$. Split $g_{j}$ as $f_{j, i}^{(2)}+f_{j, i}^{(3)}$, where

$$
f_{j, i}^{(3)}(x):=\sum_{\gamma \in \Omega_{j} \cup S_{j, 1} \cup \cdots \cup S_{j, i}} \widehat{g_{j}}(\gamma) \overline{\gamma(x)} .
$$

If

$$
\sup _{\gamma} \sum_{\gamma^{\prime} \in \gamma+U_{j}}\left|\widehat{f_{j, i}^{(2)}}\left(\gamma^{\prime}\right)\right| \leqslant \epsilon^{2}
$$

then STOP. Otherwise, there is a $\gamma_{j, i+1}$ with

$$
\sum_{\gamma^{\prime} \in \gamma_{j, i+1}+U_{j}}\left|\widehat{f_{j, i}^{(2)}}\left(\gamma^{\prime}\right)\right| \geqslant \epsilon^{2} .
$$

Set $\operatorname{STEP}=(j, i+1), S_{j, i+1}:=\left(\gamma_{j, i+1}+U_{j}\right) \backslash\left(\Omega_{j} \cup S_{j, 1} \cup \cdots \cup S_{j, i}\right)$. An important point to note is that

$$
\sum_{\gamma^{\prime} \in S_{j, i+1}}\left|\widehat{f_{j, i}^{(2)}}\left(\gamma^{\prime}\right)\right| \geqslant \epsilon^{2}:
$$

although this is ostensibly a stronger statement than (4.13), this is merely an illusion since by construction the support of $\widehat{f_{j, i}^{(2)}}$ is disjoint from $\Omega_{j} \cup S_{j, 1} \cup \cdots \cup S_{j, i}$.

Claim 2. The algorithm terminates, and in fact we have the bound $\left|\Gamma_{l}\right| \leqslant 2 \epsilon^{-2}$, independently of $l$.

Proof. To each element $\gamma_{j, i}, j \leqslant l$ contained in $\Gamma_{l}$ is associated, by (4.14), a set $S_{j, i}$ with the property that

$$
\sum_{\gamma \in S_{j, i}}\left|\widehat{f_{j, i-1}^{(2)}}(\gamma)\right| \geqslant \epsilon^{2}
$$


This implies that

$$
2 \sum_{\gamma \in S_{j, i}}|\widehat{f}(\gamma)| \geqslant \sum_{\gamma \in S_{j, i}}|\widehat{f}(\gamma)|\left|1-\beta_{j}(\gamma)\right|=\sum_{\gamma \in S_{j, i}}\left|\widehat{f_{j, i-1}^{(2)}}(\gamma)\right| \geqslant \epsilon^{2} .
$$

Recalling that the sets $S_{j, i}$ are disjoint and that $\|\widehat{f}\|_{1} \leqslant 1$, the claim follows.

Suppose that the algorithm terminates when $\mathrm{STEP}_{1}=l$. The whole purpose of having an iteration somewhat more complicated than the one we outlined at the start of the section is that we can beat the crude bound $l \leqslant 2 \epsilon^{-2}$ which follows from Claim 2 .

Claim 3. We have $l \leqslant 2 / \epsilon$.

Proof. Set $S_{j}=\bigcup_{i} S_{j, i}$. For any $j$ we have

$$
2 \sum_{\gamma \in S_{j}}|\widehat{f}(\gamma)| \geqslant \sum_{\gamma \in S_{j}}|\widehat{f}(\gamma)|\left|1-\widehat{\beta}_{j}(\gamma)\right|=\sum_{\gamma \in S_{j}}\left|\widehat{g_{j}}(\gamma)\right| \geqslant \epsilon .
$$

Since the sets $S_{j}$ are disjoint and $\|\widehat{f}\|_{1} \leqslant 1$, we do indeed have $l \leqslant 2 / \epsilon$.

To conclude the proof of Lemma 4.3, suppose that $\operatorname{STEP}=(l, m)$ at termination. By construction the decomposition

$$
f=f_{l, m}^{(1)}+f_{l, m}^{(2)}+f_{l, m}^{(3)}
$$

satisfies all of the properties required. It remains to check the bounds claimed. This is a simple matter; using the crude estimate $\eta_{j+1} \geqslant \epsilon^{5} \eta_{j}^{\prime} / 2^{11} \geqslant \epsilon^{9} \eta_{j} / 2^{21}$, we do indeed have $\eta_{l} \geqslant\left(\epsilon^{9} / 2^{21}\right)^{2 / \epsilon}$.

In order to apply Lemma 4.3, we must be able to exhibit a value of $x$ for which $\left|f^{(1)}(x)\right|$ is small. This is where we invoke a kind of "discrete intermediate value theorem" of the type used in $₫ 1$. This argument, as the reader will appreciate, is specific to the case $G=\mathbb{Z} / p \mathbb{Z}$.

Lemma 4.4. Let $f: \mathbb{Z} / p \mathbb{Z} \rightarrow \mathbb{R}$ be a function with $\mathbb{E}_{x} f(x)=0$. Let $B=B(\Gamma, \eta)$ be a regular Bohr set with normalised cutoff $\beta$, and suppose that $f^{(1)}=f * \beta$. Suppose that $\left(\epsilon^{2} \eta / 200 d\right)^{d} p>1$. Then $\min \left|f^{(1)}(x)\right| \leqslant 2 \epsilon$.

Proof. We invoke Lemma 4.1, or rather the proof of it, which tells us that if $\eta^{\prime}=$ $\epsilon^{2} \eta / 200 d$ and if $t \in B\left(\Gamma, \eta^{\prime}\right)$ then

$$
\left|f^{(1)}(x+t)-f^{(1)}(x)\right| \leqslant 2 \epsilon
$$

for any $x$. The conditions of the lemma, together with the simple bound of Lemma 3.1 (i), imply that $\left|B\left(\Gamma, \eta^{\prime}\right)\right|>1$, so there is some $t \in B\left(\Gamma, \eta^{\prime}\right), t \neq 0$. The elements $\{0, t, 2 t, 3 t, \ldots,(p-1) t\}$ are in fact just the elements of $\mathbb{Z} / p \mathbb{Z}$, listed once each. Since $\mathbb{E}_{x} f^{(1)}(x)=0$, there must be a value of $j$ for which $f^{(1)}(j t)$ and $f^{(1)}((j+1) t)$ have opposing signs. For this value of $j$, (4.15) guarantees that $\left|f^{(1)}(j t)\right| \leqslant 2 \epsilon$.

Proof of Theorem 1.3. Lemma 4.3 tells us that there is a set $\Gamma, d:=|\Gamma| \leqslant 2 / \epsilon^{2}$, and an $\eta \geqslant\left(\epsilon^{9} / 2^{21}\right)^{2 / \epsilon}$ for which the hypotheses of Lemma 4.2 are satisfied. If in addition $\min _{x}\left|f^{(1)}(x)\right| \leqslant 2 \epsilon$, then the conclusion of that lemma implies that $\min _{x}|f(x)| \leqslant 8 \epsilon$. By Lemma 4.4 this will be the case if $\left(\epsilon^{2} \eta / 200 d\right)^{d} p>1$, which will be satisfied if $\epsilon=C(\log p / \log \log p)^{1 / 3}$ for suitable $C$. 
It is a simple matter to deduce Theorem 1.2 from Theorem 1.3. Recall that $S(p)$ is the smallest possible value of $\left\|\widehat{1_{A}}\right\|_{1}$, where $A \subseteq \mathbb{Z} / p \mathbb{Z}$ is a set with cardinality $\lfloor p / 2\rfloor$.

Proof of Theorem 1.2. Set $f(x):=\left(21_{A}(x)-1\right) /\left\|\left(21_{A}-1\right)^{\wedge}\right\|_{1}$, so that $\|\widehat{f}\|_{1}=1$, and apply Theorem 1.3. One obtains

$$
\left\|\left(21_{A}-1\right)^{\wedge}\right\|_{1}^{-1}=\min _{x}|f(x)|=O(\log \log p / \log p)^{1 / 3} .
$$

Now

which means that

$$
\left(21_{A}-1\right)^{\wedge}(r)= \begin{cases}2 \widehat{1_{A}}(r) & \text { if } r \neq 0 \\ O(1 / p) & \text { if } r=0\end{cases}
$$

$$
\left\|\left(21_{A}-1\right)^{\wedge}\right\|_{1} \geqslant 2\left\|\widehat{1_{A}}\right\|_{1}+O(1) .
$$

The result follows immediately.

To conclude this section we give the proof of Theorem 1.5 and hence, by the remarks in \$1, answer the question of Gowers.

Proof of Theorem 1.5. Consider the functions

$$
\begin{aligned}
f_{0}(x) & :=f(x)-\mathbb{E} f \\
g_{0}(x) & :=g(x)-\mathbb{E} g
\end{aligned}
$$

and

$$
h(x):=f * g-\mathbb{E} f \mathbb{E} g .
$$

It is easy to see that $\mathbb{E} f_{0}=\mathbb{E} g_{0}=0$, that $\left\|f_{0}\right\|_{2},\left\|g_{0}\right\|_{2} \leqslant 1$, and that

$$
h=f_{0} * g_{0} .
$$

In particular we have, by the Cauchy-Schwarz inequality and Parseval's identity, that

$$
\|\widehat{h}\|_{1} \leqslant\left\|f_{0}\right\|_{2}\left\|g_{0}\right\|_{2} \leqslant 1
$$

(this is a special case of Young's inequality). The result follows immediately from Theorem 1.3.

\section{The Proof of Theorem 1.6}

Lemma 5.1. For any prime $p$ there is a function $F: \mathbb{Z} / p \mathbb{Z} \rightarrow \mathbb{R}$ such that $\mathbb{E} F=0$, $\|\widehat{F}\|_{1} \leqslant 1, \widehat{F}(r)=\widehat{F}(-r) \geqslant 0$ for all $r \in \mathbb{Z} / p \mathbb{Z}$, and $\min _{x}|F(x)| \gg(\log p)^{-1}$.

Proof. We may, of course, assume that $p$ is sufficiently large. Consider to begin with the very simple function

$$
g_{1}(x):= \begin{cases}1 & \text { if }|x / p|<1 / 4 \\ -1 & \text { otherwise }\end{cases}
$$

We have $\mathbb{E} g_{1}=O(1 / p)$, whilst the Fourier transform $\widehat{g}_{1}(r)$ satisfies $\widehat{g}_{1}(r)=\widehat{g}_{1}(-r)$ and

$$
\left|\widehat{g}_{1}(r)\right| \leqslant \frac{1}{p|\sin (\pi r / p)|} \leqslant \frac{1}{2|r|}
$$

for $|r|<p / 2$. For suitable $C$, the function $F_{1}(x):=g_{1}(x) / C \log p$ satisfies all the requirements of the lemma except for the condition that $\widehat{F}_{1}(r)$ be non-negative (and the 
condition that $\mathbb{E} F_{1}=0$, though this can be achieved by a very small perturbation of $\left.F_{1}\right)$.

To construct a function with non-negative Fourier transform, we begin by considering an auxilliary function on $\mathbb{R} / \mathbb{Z}$. Define

$$
f_{1}(\theta):=-2 \log |2 \sin \pi \theta|
$$

for $\theta \neq 0$, and $f_{1}(0)=0$. Note that $f \in L^{p}$ for all $p \in[1, \infty)$. We claim that the Fourier transform of $f_{1}$ is given by

$$
\widehat{f}_{1}(m)= \begin{cases}1 /|m| & \text { if } m \in \mathbb{Z} \backslash\{0\} \\ 0 & \text { otherwise. }\end{cases}
$$

Heuristically, this can be seen by observing the Taylor expansion

$$
-\log (1-z)=z+\frac{z^{2}}{2}+\frac{z^{3}}{3}+\ldots
$$

setting $z=e^{2 \pi i \theta}$ and $z=e^{-2 \pi i \theta}$, and adding. To justify the assertion rigourously, one could proceed as follows. Using Cauchy's formula for derivatives, one confirms that

$$
\frac{1}{2 \pi i} \int_{\Gamma(\epsilon)} \frac{\log (1-z)}{z^{n+1}} d z=-\frac{1}{n}
$$

for $n \geqslant 1$, where $\Gamma(\epsilon)$ is the contour consisting of the circle $|z|=1$ indented to the left near $z=1$ using a semicircle of radius $\epsilon$. The contribution from the semicircle of radius $\epsilon$ is $O(\epsilon \log (1 / \epsilon))$, which tends to 0 as $\epsilon \rightarrow 0$. The remainder of the integral is

$$
\int_{\theta \in \mathbb{R} / \mathbb{Z}:|\theta| \geqslant \epsilon} \log \left(1-e^{2 \pi i \theta}\right) e^{-2 \pi i n \theta} d \theta
$$

This differs from the integral over all $\theta \in \mathbb{R} / \mathbb{Z}$ by an error of

$$
O\left(\int_{0}^{\epsilon}|\log \theta| d \theta\right)
$$

which is $O(\epsilon \log (1 / \epsilon))$. Letting $\epsilon \rightarrow 0$ in (5.3) , then, we see that

$$
\int_{\theta \in \mathbb{R} / \mathbb{Z}} \log \left(1-e^{2 \pi i \theta}\right) e^{-2 \pi i n \theta} d \theta=-\frac{1}{n}
$$

for $n \geqslant 1$. By a similar argument, this integral vanishes when $n \leqslant 0$. Furthermore by an almost identical argument one may confirm that

$$
\int_{\mathbb{R} / \mathbb{Z}} \log \left(1-e^{-2 \pi i \theta}\right) e^{-2 \pi i n \theta}= \begin{cases}1 / n & \text { if } n \leqslant-1 \\ 0 & \text { otherwise. }\end{cases}
$$

Adding this to (5.4) establishes the claim (5.2).

Now $f_{1}$ is useless to us as it stands, since $\widehat{f}_{1}$ does not lie in $l^{1}(\mathbb{Z})$. We must also transfer $f_{1}$ to a function defined on $\mathbb{Z} / p \mathbb{Z}$. We modify $f_{1}$ by defining

$$
f_{2}:=f_{1} * \chi * \chi
$$

where

$$
\chi(\theta)=\frac{C p}{2} 1_{|\theta| \leqslant 1 / C p}(\theta)
$$


for some large constant $C$ to be specified later. Note that

$$
\widehat{f}_{2}(m)=\widehat{f}_{1}(m)\left(\frac{C p \sin 2 \pi m / C p}{2 \pi m}\right)^{2}
$$

where the bracketed expression is to be interpreted as 1 if $m=0$. Consider the function $g_{2}: \mathbb{Z} / p \mathbb{Z} \rightarrow \mathbb{R}$ defined by

$$
g_{2}(x)=\sum_{m \in \mathbb{Z}} \widehat{f}_{2}(m) e^{2 \pi i m x / p}
$$

Since $\widehat{f}_{2} \in l^{1}(\mathbb{Z})$, the series converges uniformly and

$$
g_{2}(x)=f_{2}(x / p)
$$

provided that $x \neq 0$.

Now we have

$$
\left|f_{1}^{\prime}(\theta)\right|=|2 \pi \cot \pi \theta| \leqslant \frac{2}{|\theta|}
$$

for all $0<|\theta| \leqslant 1 / 2$. From the definition (5.5), it follows that

$$
\left|g_{2}(x)-f_{1}(x / p)\right| \leqslant \frac{2}{C p} \sup _{|\theta-x / p| \leqslant 2 / p C}\left|f_{1}^{\prime}(\theta)\right| \leqslant 8 / C
$$

provided that $x \neq 0$ and $C \geqslant 4$.

All we need to know about $g_{2}(0)$ is that it is large, to which end the bound

$$
g_{2}(0) \geqslant g_{2}(1)
$$

will be quite sufficient.

The Fourier transform $\widehat{g}_{2}(r)$ on $\mathbb{Z} / p \mathbb{Z}$ is given by

$$
\widehat{g}_{2}(r)=\sum_{\substack{m \in \mathbb{Z} \\ m \equiv r(\bmod p)}} \widehat{f}_{2}(m)
$$

(this is an instance of the Poisson summation formula, but in this case it follows from the inversion formula

$$
g_{2}(x)=\sum_{r} \widehat{g}_{2}(r) e^{2 \pi i r x / p}
$$

upon comparing with (5.7) and recalling the uniqueness of Fourier expansion). In particular $\widehat{g}_{2}(r)=\widehat{g}_{2}(-r)$. Now $\widehat{f}_{2}(m)$ is real and non-negative, and hence so is $\widehat{g}_{2}(r)$ and we have

$$
\widehat{g}_{2}(r) \geqslant \widehat{f}_{2}(\bar{r})
$$

where $\bar{r}$ is the unique integer with $|\bar{r}|<p / 2$ and $\bar{r} \equiv r(\bmod p)$. Using the inequality $\sin \theta / \theta \geqslant 1-\theta^{2} / 6$ and (5.2), we thus have

$$
\widehat{g}_{2}(r) \geqslant \widehat{f}_{1}(\bar{r})\left(1-\frac{2 \pi^{2}|\bar{r}|^{2}}{3 C^{2} p^{2}}\right)^{2} \geqslant \frac{1}{|\bar{r}|}\left(1-\frac{4}{C^{2}}\right)
$$

for $r \neq 0$. 
In the other direction, we have the estimate

$$
\left\|\widehat{g}_{2}\right\|_{1}=\sum_{m} \widehat{f}_{2}(m)=\sum_{\substack{m \in \mathbb{Z} \\ m \neq 0}} \frac{1}{|m|}\left(\frac{C p \sin 2 \pi m / C p}{2 \pi m}\right)^{2} \leqslant 2 \sum_{m=1}^{\infty} \min \left(\frac{1}{m}, \frac{C^{2} p^{2}}{4 \pi^{2} m^{3}}\right) \ll \log p .
$$

Now define

$$
g_{3}:=g_{1}+g_{2}
$$

From (5.1) and (5.11), we see that $\widehat{g}_{3}(r)=\widehat{g}_{3}(-r)$, and that $\widehat{g}_{3}(r) \geqslant 0$ for all $r \neq 0$, provided that $C^{2} \geqslant 8$. From (5.1) and (5.12) we see that

$$
\left\|\widehat{g}_{3}\right\|_{1} \ll \log p \text {. }
$$

We claim that if $C \geqslant 1000$ then

$$
\left|g_{3}(x)\right| \geqslant 1 / 4 \quad \text { for all } x .
$$

To see this, observe from (5.8) that if $1 / 4<|x / p| \leqslant 1 / 2$ then

$$
g_{3}(x)=-1+g_{2}(x) \leqslant-1+f_{1}(x / p)+8 / C \leqslant-1-\log 2+8 \pi / C \leqslant-1 .
$$

If $0<|x / p|<1 / 4$ then we have

$$
g_{3}(x)=1+g_{2}(x) \geqslant 1+f_{1}(x / p)-8 / C \geqslant 1-\log 2-8 \pi / C \geqslant 1 / 4 .
$$

Finally from (5.9) we have

$$
g_{3}(0)=1+g_{2}(0) \geqslant 1+g_{2}(1 / p) \geqslant 1+f_{1}(1 / p)-8 / C \geqslant 1 / 4 .
$$

This proves the claim.

The function $F$ (whose construction is our goal) will essentially be a rescaled version of $g_{3}$, but it is required to satisfy $\mathbb{E} F=0$. To this end we note that

$$
\mathbb{E} g_{1}=O(1 / p)
$$

whilst, from (5.10), we have

$$
\mathbb{E} g_{2}=\sum_{m \equiv 0(\bmod p)} \widehat{f}_{2}(m) \leqslant 2 \sum_{\substack{m=1 \\ m \equiv 0(\bmod p)}}^{\infty} \min \left(\frac{1}{m}, \frac{C^{2} p^{2}}{4 \pi^{2} m^{3}}\right)=O(1 / p) .
$$

Thus $\mathbb{E} g_{3}=O(1 / p)$, and so if we define

$$
g_{4}:=g_{3}-\mathbb{E} g_{3}
$$

then we still have

$$
\left|g_{4}(x)\right| \geqslant 1 / 4+O(1 / p) \geqslant 1 / 5
$$

for $p$ sufficiently large. Of course, since $\widehat{g}_{4}(r)=\widehat{g}_{3}(r)$ for $r \neq 0$, we also have $\widehat{g}_{4}(r)=$ $\widehat{g}_{4}(-r) \geqslant 0$.

At last we may define $F$ by

$$
F(x):=g_{4}(x) /\left\|\widehat{g}_{4}\right\|_{1} .
$$

The asserted properties of $F$ are immediate from the definition, the facts we have assembled about $g_{4}$, and (5.13).

We conclude this section by proving Theorem [1.6, which asserted the existence of a function $f: \mathbb{Z} / p \mathbb{Z} \rightarrow \mathbb{R}$ with $\mathbb{E} f=0,\|f\|_{2} \leqslant 1$, and $\left|f * f^{\circ}(x)\right| \geqslant c / \log p$ for all $x$. 
Proof of Theorem 1.6. Let $F$ be the function constructed in Lemma 5.1. Take the function $f$ with $\widehat{f}=\sqrt{\widehat{F}}$. Then

$$
\begin{gathered}
\mathbb{E} f=\widehat{f}(0)=\sqrt{\widehat{F}(0)}=\sqrt{\mathbb{E} F}=0, \\
\mathbb{E} f^{2}=\sum_{r} \widehat{f}(r)^{2}=\|\widehat{F}\|_{1} \leqslant 1,
\end{gathered}
$$

and

$$
f * f^{\circ}=F
$$

The theorem follows.

\section{An eXAmple For Gowers' QUeStion}

In this section we prove Theorem 1.7, which asserted that there is a set $A \subseteq \mathbb{Z} / p \mathbb{Z}$ with $|A|=\lfloor p / 2\rfloor$ and

$$
|A \cap(A+x)-p / 4| \geqslant c / \log p \log \log p
$$

for all $x$. Noting that

$$
|A \cap(A+x)-p / 4|=p f * f^{\circ}(x),
$$

where $f:=1_{A}-1 / 2$, we see that this is a matter of finding a function $f$ in Theorem 1.6 which somehow "resembles a set". The function $f$ constructed at the end of $\$ 5$ need not have this property. Note, however, that we could have exercised considerable freedom in our choice of $f$ : any function such that $\widehat{f}(r)=\xi_{r} \sqrt{\widehat{F}(r)}$, where $\xi_{r} \in\{-1,1\}$, would have done. By choosing the signs $\xi_{r}$ at random we may force $f$ to behave well in $L^{\infty}$, and hence to resemble a set. The rest of the section is devoted to the details of such an argument.

Suppose, then, that $\xi=\left\{\xi_{r} \in\{ \pm 1\}, 0 \leqslant r<p / 2\right\}$ is a sequence of independent random signs and that $f=f_{\xi}$ is the function whose Fourier transform satisfies $\widehat{f}(r)=\xi_{r} \sqrt{\widehat{F}(r)}$, $0 \leqslant r<p / 2$, and $\widehat{f}(r)=\widehat{f}(-r)$.

Let us reiterate the properties enjoyed by $f_{\xi}$ : It satisfies $\mathbb{E} f_{\xi}=0,\left\|f_{\xi}\right\|_{2} \leqslant 1$, and $\left|f_{\xi} * f_{\xi}^{\circ}(x)\right| \gg 1 / \log p$ for all $x$.

Lemma 6.1. There is an absolute constant $c$ and a choice of signs $\xi$ such that

$$
\mathbb{E}_{x \in \mathbb{Z} / p \mathbb{Z}} \exp \left(c f_{\xi}(x)^{2}\right) \ll 1 .
$$

Proof. Let $\left(a_{r}\right)_{0 \leqslant r<p / 2}$ be any sequence of real numbers. It is well-known (a simple instance of Khintchine's inequality, see for example [7]) that

$$
\mathbb{E}_{\xi}\left|\sum_{r} a_{r} \xi_{r}\right|^{2 k} \leqslant\left(C_{0} k \sum_{r} a_{r}^{2}\right)^{k}
$$

for some absolute $C_{0}$. It follows that for any $c \geqslant 0$ we have

$$
\mathbb{E}_{\xi} \exp \left(c\left(\sum_{r} a_{r} \xi_{r}\right)^{2}\right) \leqslant \sum_{k \geqslant 0} \frac{1}{k !}\left(c C_{0} k \sum_{r} a_{r}^{2}\right)^{k} .
$$


Now for any $x \in \mathbb{Z} / p \mathbb{Z}$ we have

$$
f_{\xi}(x)=\sum_{0<r<p / 2} 2 \xi_{r} \sqrt{\widehat{F}(r)} \cos (2 \pi x r / p) .
$$

For each fixed $x$ this has the form

$$
\sum_{r} a_{r} \xi_{r}
$$

where

$$
a_{r}=2 \sqrt{\widehat{F}(r)} \cos (2 \pi x r / p)
$$

Since $\|\widehat{F}\|_{1} \leqslant 1$, we have

$$
\sum_{r} a_{r}^{2} \leqslant 4
$$

In view of (6.2) this means that

$$
\mathbb{E}_{\xi} \exp \left(c f_{\xi}(x)^{2}\right) \leqslant \sum_{k \geqslant 0} \frac{1}{k !}\left(4 c C_{0} k\right)^{k} .
$$

By Stirling's formula this series converges provided that $4 c C_{0}<1 / e$, an inequality which can certainly be achieved by selecting $c$ sufficiently small.

Averaging over $x$, we see that

$$
\mathbb{E}_{x} \mathbb{E}_{\xi} \exp \left(c f_{\xi}(x)^{2}\right) \ll 1 .
$$

In particular there is at least one choice of the signs $\xi$ for which the claimed inequality is true.

The next lemma is a technical fact used in the proof of Lemma 6.3 below.

Lemma 6.2. Let $f_{1}, f_{2}: \mathbb{Z} / p \mathbb{Z} \rightarrow \mathbb{R}$ be two functions. Then the following inequality holds:

$$
\left\|f_{1} * f_{1}^{\circ}-f_{2} * f_{2}^{\circ}\right\|_{\infty} \leqslant\left\|f_{1}-f_{2}\right\|_{2}\left(\left\|f_{1}\right\|_{2}+\left\|f_{2}\right\|_{2}\right) .
$$

Proof. By the Cauchy-Schwarz inequality we have

$$
|f * g(x)| \leqslant\|f\|_{2}\|g\|_{2}
$$

for any two functions $f, g$ and for any $x \in \mathbb{Z} / p \mathbb{Z}$. Setting $g:=f_{1}-f_{2}$ we have

$$
\left|\left(f_{1} * f_{1}^{\circ}\right)(x)-\left(f_{2} * f_{2}^{\circ}\right)(x)\right| \leqslant\left|f_{2} * g^{\circ}(x)\right|+\left|g * f_{1}^{\circ}(x)\right| \leqslant\left\|f_{2}\right\|_{2}\|g\|_{2}+\left\|f_{1}\right\|_{2}\|g\|_{2},
$$

as required.

The next lemma is a stronger version of Theorem 1.6.

Lemma 6.3. There is a function $f: \mathbb{Z} / p \mathbb{Z} \rightarrow \mathbb{R}$ such that $\mathbb{E} f=0,\|f\|_{2} \ll 1, \mid f *$ $f^{\circ}(x) \mid \gg 1 / \log p$ for all $x$, and with the additional property that $\|f\|_{\infty} \ll \sqrt{\log \log p}$.

Proof. Take a function $f_{\xi}$ satisfying the conclusion of Lemma 6.1, that is to say for which the inequality

$$
\mathbb{E}_{x} \exp \left(c f_{\xi}(x)^{2}\right) \ll 1
$$

is satisfied. Let $C$ be a large absolute constant, and consider the set

$$
A:=\left\{x:\left|f_{\xi}(x)\right| \geqslant C \sqrt{\log \log p}\right\} .
$$


It follows from (6.4) that, for a suitable choice of $C$, we have

$$
\mathbb{E} 1_{A} \ll(\log p)^{-10} .
$$

Choose an arbitrary set $A^{\prime}$ such that $A \subseteq A^{\prime}$ and

$$
(\log p)^{-10} \ll \mathbb{E} 1_{A^{\prime}} \ll(\log p)^{-10} .
$$

Define $f$ by

$$
f(x):= \begin{cases}f_{\xi}(x) & \text { if } x \notin A^{\prime} ; \\ \kappa & \text { if } x \in A^{\prime},\end{cases}
$$

where $\kappa$ is selected so that $\mathbb{E} f=0$.

Observe that

$$
|\kappa|=\left|\mathbb{E}_{x \in A^{\prime}} f_{\xi}(x)\right| \leqslant \mathbb{E}_{x \in A^{\prime}}\left|f_{\xi}(x)\right| .
$$

From (6.4) one may deduce without difficulty that

$$
\mathbb{E}_{x \in S}\left|f_{\xi}(x)\right| \ll\left(\log \left(1 / \mathbb{E} 1_{S}\right)\right)^{1 / 2}
$$

for any nonempty set $S \subseteq \mathbb{Z} / p \mathbb{Z}$. Hence, in view of (6.6), we have the bound

$$
|\kappa| \ll \sqrt{\log \log p}
$$

This immediately implies that $\|f\|_{\infty} \ll \sqrt{\log \log p}$.

To obtain the upper bound on $\|f\|_{2}$, it suffices to note that

$$
\left\|f-f_{\xi}\right\|_{2}=\left\|\left(f_{\xi}-\kappa\right) 1_{A}\right\|_{2} \ll \sqrt{\log p}\left(\mathbb{E} 1_{A}\right)^{1 / 2} \ll(\log p)^{-4} .
$$

Furthermore, this inequality together with Lemma 6.2 implies that

$$
\left\|f * f^{\circ}-f_{\xi} * f_{\xi}^{\circ}\right\|_{\infty} \ll(\log p)^{-4} .
$$

In view of the fact that

$$
\left|f_{\xi} * f_{\xi}^{\circ}(x)\right| \gg 1 / \log p
$$

for all $x \in \mathbb{Z} / p \mathbb{Z}$, we obtain the desired lower bound

$$
\left|f * f^{\circ}(x)\right| \gg 1 / \log p .
$$

Remark. A well-known conjecture of Komlós implies that if $\left(a_{i j}\right)_{1 \leqslant i, j \leqslant n}$ are any scalars then there is a choice of signs $\xi_{j}$ so that

$$
\left|\sum_{j} a_{i j} \xi_{j}\right| \leqslant C\left(\sum_{j} a_{i j}^{2}\right)^{1 / 2},
$$

for some absolute constant $C$. This conjecture, if true, would allow us to replace the $\sqrt{\log \log p}$ appearing in Lemma 6.3 by an absolute constant, and to remove all subsequent appearances of $\log \log p$. The best result towards Komlós' conjecture is due to Banaszczyk [1]; it may be that his ideas may be applied in our context to reduce the $\log \log p$ factors to something a little smaller, but it does not seem worth the effort of pursuing this line of inquiry.

Using a simple linear transformation, Lemma 6.3 has the following consequence.

Lemma 6.4. There exists a function $f: \mathbb{Z} / p \mathbb{Z} \rightarrow[0,1]$ such that $\mathbb{E} f=1 / 2$ and

$$
\left|f * f^{\circ}(x)-1 / 4\right| \gg 1 / \log p \log \log p
$$

for all $x \in \mathbb{Z} / p \mathbb{Z}$. 
To complete the proof Theorem 1.7 we use probabilistic arguments again, in a fairly standard manner. We choose a set $B$ by selecting each $x \in \mathbb{Z} / p \mathbb{Z}$ to lie in $B$ independently at random with probability $f(x)$, and then show that with high probability $|B|$ is close to $p / 2$ and $1_{B} * 1_{B}^{\circ}(x)$ is close to $f * f^{\circ}(x)$ whenever $x \neq 0$. We then modify $B$ by adding or deleting a small number of elements to give a set $A$ with $|A|=\lfloor p / 2\rfloor$.

For each $x \in \mathbb{Z} / p \mathbb{Z}$ we write $X_{x}$ for the random variable which is 1 with probability $f(x)$ and 0 with probability $1-f(x)$. To prove the statements we need, it is natural to use one of the standard large-deviation type inequalities. Amongst inequalities of this sort, one particularly suited to our purpose is Hoeffding's inequality (see, for example, 4]).

Lemma 6.5 (Hoeffding's inequality). Suppose that $Y_{1}, \ldots, Y_{m}$ are independent random variables such that $\left|Y_{i}\right| \leqslant 1$ for each $i$. Write $Y:=m^{-1}\left(Y_{1}+\cdots+Y_{m}\right)$ and $\mu:=\mathbb{E} Y$. Then for any $t \geqslant 0$ we have

$$
\mathbb{P}(|Y-\mu| \geqslant t) \leqslant 2 e^{-m t^{2} / 2} .
$$

It follows immediately from this, applied with $m=p$ and $Y_{x}=X_{x}$, that

$$
\mathbb{P}\left(|| B|-p / 2| \geqslant p^{2 / 3}\right) \ll e^{-p^{1 / 3} / 2} .
$$

Fix $x \in \mathbb{Z} / p \mathbb{Z}, x \neq 0$, and look at the expression

$$
1_{B} * 1_{B}^{\circ}(x)=p^{-1} \sum_{y} X_{y} X_{y-x} .
$$

Writing

we have

$$
Z_{y}:=X_{y} X_{y-x}
$$

$$
p^{-1} \sum_{y} \mathbb{E} Z_{y}=f * f^{\circ}(x)
$$

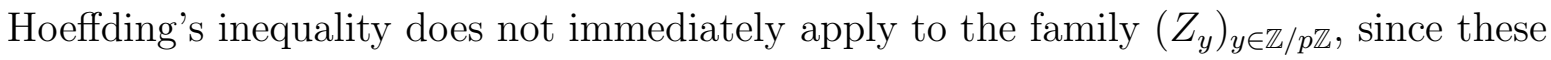
random variables are not independent. However we may partition the family into three subfamilies $\left(Z_{y}\right)_{y \in S_{i}}, i=1,2,3$, where $\left|S_{i}\right| \geqslant p / 5$, and within each family the random variables $Z_{y}$ are jointly independent. This may be achieved by forming the graph on vertex set $\mathbb{Z} / p \mathbb{Z}$ in which verex $i$ is joined to vertex $j$ if $i-j=x$; this is a cycle of length $p$, and we partition its vertex set into 3 classes each having size at least $p / 5$.

Hoeffding's inequality now applies, and we have

$$
\mathbb{P}\left(\left.|| S_{i}\right|^{-1} \sum_{y \in S_{i}} Z_{y}-\left|S_{i}\right|^{-1} \sum_{y \in S_{i}} \mathbb{E} Z_{y} \mid \geqslant t\right) \leqslant 2 e^{-p t^{2} / 10}
$$

for $i=1,2,3$. It follows using the triangle inequality that

$$
\mathbb{P}\left(\left|p^{-1} \sum_{y \in \mathbb{Z} / p \mathbb{Z}} Z_{y}-p^{-1} \sum_{y \in \mathbb{Z} / p \mathbb{Z}} \mathbb{E} Z_{y}\right| \geqslant t\right) \leqslant 6 e^{-p t^{2} / 10},
$$

that is to say

$$
\mathbb{P}\left(\left|\left(1_{B} * 1_{B}^{\circ}\right)(x)-\left(f * f^{\circ}\right)(x)\right| \geqslant t\right) \leqslant 6 e^{-p t^{2} / 10} .
$$

Taking $t=p^{-1 / 3}$, we see from this and (6.8) that with probability close to 1 we have both

$$
|| B|-p / 2| \leqslant p^{2 / 3}
$$


and

$$
\left|1_{B} * 1_{B}^{\circ}(x)-f * f^{\circ}(x)\right| \leqslant p^{-1 / 3}
$$

for all $x \neq 0$. Fix a specific set $B$ verifying (6.9) and (6.10). Form a set $A$ by adding to or deleting from $B$ arbitrary elements so that $|A|=\lfloor p / 2\rfloor$. In view of (6.9) we have

$$
\left\|1_{A}-1_{B}\right\|_{2} \leqslant 2 p^{-1 / 6}
$$

and hence by Lemma 6.2 we infer that

$$
\left\|1_{A} * 1_{A}^{\circ}-1_{B} * 1_{B}^{\circ}\right\|_{\infty} \leqslant 4 p^{-1 / 6} .
$$

It follows from this, (6.10) and (6.7) that

$$
\left|1_{A} * 1_{A}^{\circ}(x)-1 / 4\right| \gg 1 / \log p \log \log p
$$

for all $x \neq 0$. This inequality is manifestly true for $x=0$ as well, and this completes the proof of Theorem 1.7

\section{A RECEnt DEVELOPMENT}

We have recently learnt that Sanders 11 has combined some of our methods with some new ideas, and has been able to improve the bound in Theorem 1.3 to $(\log p)^{-1 / 2+\epsilon}$.

\section{REFERENCES}

[1] W. Banaszczyk, Balancing vectors and Gaussian measures of $n$-dimensional convex bodies, Random Structures Algorithms 12 (1998), no. 4, 351-360.

[2] J. Bourgain, On triples in arithmetic progression, GAFA 9 (1999), no. 5, 968-984.

[3] H. T. Croft, Some Problems, Eureka 1968.

[4] B. J. Green, Large deviation results for combinatorics and number theory, available at http://www .maths.bris.ac.uk/mabjg/papers/deviate.pdf

[5] B. J. Green A Szemerédi-type regularity lemma in abelian groups, GAFA 15 (2005), no. 2, 340-376.

[6] B. J. Green Finite field models in additive combinatorics, Surveys in Combinatorics 2005, LMS Lecture Notes in Math. 327, 1-27.

[7] J.-P. Kahane, Some random series of functions, D. C. Heath and Company, Lexington, Massachusetts, 1968.

[8] S. V. Konyagin, On the Littlewood problem, Izv. Akad. Nauk SSSR Ser. Mat. 45 (1981), no. 2, 243-265, 463.

[9] T. P. Lukashenko, On properties of the maximal function of a measure $\nu$ over a measure $\mu$, Math. Notes, 39 (1986), no. 2, 212-227.

[10] O. C. McGehee, L. Pigno and B. Smith, Hardy's inequality and the $L^{1}$ norm of exponential sums, Ann. of Math. (2) 113 (1981), no. 3, 613-618.

[11] T. W. Sanders, The Littlewood-Gowers problem, preprint available at http: //www . arxiv.org/abs/math. CA/0605522.

[12] T. C. Tao, The Bourgain-Roth theorem, expository note, available at http: //www.math.ucla.edu/tao/preprints/Expository/roth-bourgain.dvi

[13] T. C. Tao, Lecture notes 5 from Math 254A, available at http://www.math.ucla.edu/tao/254a.1.03w/notes5.dvi

Department of Mathematics, University of Bristol, University Walk, Bristol BS8 1TW, ENGLAND

E-mail address: b.j.green@bristol.ac.uk

Department of Mathematics and Mechanics, Moscow State University, Moscow 119992, RUSSIA

E-mail address: konyagin@ok.ru 